\title{
Wave function optimization in the variational Monte Carlo method
}

\author{
Sandro Sorella \\ International School for Advanced Studies (SISSA) Via Beirut 2,4 34014 Trieste, \\ Italy and INFM Democritos National Simulation Center, Trieste, Italy
}

(Dated: October 29, 2018)

\begin{abstract}
An appropriate iterative scheme for the minimization of the energy, based on the variational Monte Carlo (VMC) technique, is introduced and compared with existing stochastic schemes. We test the various methods for the 1D Heisenberg ring and the $2 \mathrm{D}$ t-J model and show that, with the present scheme, very accurate and efficient calculations are possible, even for several variational parameters. Indeed, by using a very efficient statistical evaluation of the first and the second energy derivatives, it is possible to define a very rapidly converging iterative scheme that, within VMC, is much more convenient than the standard Newton method. It is also shown how to optimize simultaneously both the Jastrow and the determinantal part of the wave function.
\end{abstract}

Since the seminal work of Gutzwiller, a large amount of progress has been achieved in the understanding of strongly correlated materials by means of correlated wave functions (WF). The fractional quantum Hall effect 1 and the theory-still controversial- of high temperature superconductors ${ }^{2}$ are two important among many other examples.

Most correlated WF, here indicated by $\left|\psi_{G}\right\rangle$, can be obtained 3.4 .5 by applying a two body Jastrow factor $J$ to a mean-field WF $|D\rangle$ described by a single determinant or a superconducting BCS state (SDBCS), so that:

$$
\left|\psi_{G}\right\rangle=J|D\rangle
$$

where $J$ contains two-body density-density and spin correlations. For accurate calculations, the inclusion of these two-body correlations in $\psi_{G}$ is possible by means of the VMC statistical approach.

However the application of correlated WF to complex electronic systems ${ }^{6.7 .8}$, or-e.g.-to describe correctly the metal-insulator transition ${ }^{9}$, requires the use of many variational parameters. A drawback that has certainly limited the application of VMC to important physical and chemical problems with a large number of electrons.

In order to overcome the above limitations of VMC, in this paper we introduce a minimization scheme, that allows to optimize efficiently the energy expectation value:

$$
E_{\alpha}=\frac{\left\langle\psi_{\alpha}|H| \psi_{\alpha}\right\rangle}{\left\langle\psi_{\alpha} \mid \psi_{\alpha}\right\rangle}
$$

where $H$ is the model Hamiltonian considered, $\alpha=$ $\alpha_{1}, \alpha_{2}, \cdots \alpha_{p}$ is a set of $p$ variational parameters appearing in the WF $\psi_{\alpha}(x)=\left\langle x \mid \psi_{\alpha}\right\rangle$, where $|x\rangle$ denotes a configuration with defined electron spins and positions. Henceforth the symbol $<>$ indicates the quantum expectation value over the state $\psi_{\alpha}$, so that $E_{\alpha}=\langle H\rangle$. Within the Newton method (NM), in order to improve the variational parameters, $E_{\alpha+\gamma}$ is expanded quadratically in $\gamma$ and minimized, then the parameters are changed $\alpha^{\prime}=\alpha+\gamma$, and the iteration repeated until $\gamma$ is negligible. In the following we describe an iterative technique, that is based on a similar strategy, and that, within VMC, performs much better than standard NM.
At each iteration and for each variational parameter $\alpha_{k}$ we define ${ }^{10}$ an operator $O_{k}$, diagonal in the configuration basis, with diagonal elements:

$$
O_{k}(x)=\frac{\partial_{\alpha_{k}} \psi_{\alpha}(x)}{\psi_{\alpha}(x)} .
$$

In order to simplify the derivation we assume that these operators $O_{k}$ appear in the WF in the simple exponential form $\exp \left[\sum_{k} \alpha_{k}\left(O_{k}-<O_{k}>\right)\right]$. This is certainly the case for the Jastrow factor $J$, where the operators $O_{k}$ are just density-density or spin-spin correlations. Here for convenience we have subtracted from each $O_{k}$ the corresponding average value $\left\langle O_{k}\right\rangle$, which provides an irrelevant multiplicative constant in the WF. For the variational parameters corresponding to the uncorrelated part $|D\rangle$, this exponential form is not exactly fulfilled, but is valid only for small changes of the variational parameters $\left.\left|\psi_{\alpha+\gamma}\right\rangle \propto \exp \left[\sum_{k} \gamma_{k}\left(O_{k}-<O_{k}\right\rangle\right)\right]\left|\psi_{\alpha}\right\rangle$, namely within linear order in $\gamma$. In such a case, this expression does not provide the exact second derivative of the WF with respect to $\gamma$. Although this information is in principle required for the NM, we will show that the accurate determination of these terms is not really important within VMC, because the standard NM is rather inefficient even when these second WF derivatives are computed exactly. To this purpose we write $\left|\psi_{\alpha+\gamma}\right\rangle$ in a slightly more general form that depends on a further parameter $\beta$ :

$$
\begin{aligned}
\left|\psi_{\alpha+\gamma}\right\rangle & \simeq\left[1+\sum_{k} \gamma_{k}\left(O_{k}-<O_{k}>\right)\right. \\
+ & \left.\frac{\beta}{2} \sum_{k, k^{\prime}} \gamma_{k} \gamma_{k^{\prime}}\left(O_{k}-<O_{k}>\right)\left(O_{k^{\prime}}-<O_{k^{\prime}}>\right)\right]\left|\psi_{\alpha}\right\rangle
\end{aligned}
$$

As discussed before, this expansion is valid only within linear (quadratic) order in $\gamma$ for $\beta \neq 1(\beta=1$ Jastrow case). We consider this more general form because the value of $\beta$ can be used to improve the efficiency of the minimization scheme within the VMC. On the other hand it is clear that, as the minimum is approached, the non linear contribution proportional to $\beta$, as well as the higher order ones, become negligible and irrelevant for the WF optimization. 
By substituting expression (4) in Eq.(2), the energy can be systematically expanded in powers of $\gamma_{k}$ :

$$
\Delta E=-\sum_{k} \gamma_{k} f_{k}+\frac{1}{2} \sum_{k, k^{\prime}} \gamma_{k} \gamma_{k^{\prime}}\left[S_{h}+(1+\beta) G\right]^{k, k^{\prime}}
$$

where:

$$
\begin{aligned}
S_{h}^{k, k^{\prime}} & =<\left[O_{k},\left[H, O_{k^{\prime}}\right]\right]> \\
G^{k, k^{\prime}} & =2<\left(H-E_{\alpha}\right)\left(O_{k}-<O_{k}>\right)\left(O_{k^{\prime}}-<O_{k^{\prime}}>\right)> \\
f_{k} & =-\partial_{\alpha_{k}} E_{\alpha}=-2<\left(H-E_{\alpha}\right) O_{k}>
\end{aligned}
$$

In the above equations we have used the hermitian character of all the operators involved, implying for instance that $\left\langle O_{k} H\right\rangle=\left\langle H O_{k}\right\rangle ; f_{k}$ indicate the forces acting on the variational parameters and vanishing at the minimum energy condition, $S_{h}^{k, k^{\prime}}$ represent the excitation matrix elements corresponding to the operators $O_{k}, G^{k, k^{\prime}}$ take into account the remaining contributions appearing when the WF is not exact $\left.\left(<H-E_{\alpha}\right\rangle \neq 0\right)$, whereas the square brackets indicate the commutator. The WF parameters can be then iteratively changed $\alpha_{k} \rightarrow \alpha_{k}+\gamma_{k}$, by minimizing Eq. (15) whenever $S_{h}+(1+\beta) G$ is positive definite. The minimum energy is obtained for:

$$
\begin{aligned}
\gamma & =B^{-1} \mathbf{f} \quad \text { with }: \\
B & =S_{h}+(1+\beta) G
\end{aligned}
$$

If $B$ is not positive definite, the quadratic form (5) is not bounded from below, meaning that higher order terms are important in the expansion (5). In this case the correction (8) may lead to a higher energy rather than to a lower energy WF. In order to overcome this difficulty, we change the matrix $B \rightarrow B+\mu S$ where $S$ is the positive definite $\underline{\underline{11}}$ overlap matrix:

$$
S^{k, k^{\prime}}=<\left(O_{k}-<O_{k}>\right)\left(O_{k^{\prime}}-<O_{k^{\prime}}>\right)>
$$

Similarly to the previous Stochastic Reconfiguration technique ${ }^{10}$, we use the same matrix $S$ in order to have a well defined minimum for the energy $\Delta E$ in all cases. This is achieved by imposing the constraint that the linear WF change $\Delta \mathrm{WF}=\left(\left|\psi_{\alpha+\gamma}\right\rangle-\left|\psi_{\alpha}\right\rangle\right) /\left|\psi_{\alpha}\right|$, obtained with $\beta=0$ in Eq4 cannot be larger than a certain amount $r$. Hence the control parameter $r$ is defined by means of the inequality:

$$
|\Delta \mathrm{WF}|^{2} \leq r^{2}
$$

where, from Eqs. (410), $|\Delta \mathrm{WF}|^{2}=\sum_{k, k^{\prime}} \gamma_{k} \gamma_{k^{\prime}} S^{k, k^{\prime}}$. This constraint allows to work always with a positive definite matrix $B$ and, for small enough $r$, the energy is certainly lowered by changing the parameters according to (8). The constant $\mu \geq 0$ is non zero whenever (9) is non positive definite or $|\Delta \mathrm{WF}|$ corresponding to (8) exceeds $r$. In these cases the constant $\mu$ is obtained as a Lagrange multiplier, namely by minimizing $\Delta E+\mu|\Delta \mathrm{WF}|^{2}$, with the condition $|\Delta \mathrm{WF}|=r$, which is simple to fulfill with standard iterative schemes. A similar control parameter was used in Ref $[6$, by adding the identity matrix $I$ scaled by $a_{\text {diag }}>0$ to the Hessian, namely $B=S_{h}+2 G+a_{\text {diag }} I$, so that for $a_{\text {diag }} \rightarrow \infty$ the steepest descent is obtained, whereas within our scheme, for $\mu \rightarrow \infty$ the stochastic reconfiguration (SR) technique is recovered with $B=S / \Delta t$ and $\Delta t=1 / \mu$ small 10

As it is clear from its definition (7) the matrix $G$ is zero when $\left\langle\psi_{\alpha}\right|$ coincides with the exact ground state, because in this case $\left\langle\psi_{\alpha}\right|\left(H-E_{\alpha}\right)=0$. Therefore this matrix should be very small compared with $S_{h}$ for a good variational WF. This implies that, by changing $\beta$ in Eq9 we can obtain iterative methods converging to the minimum energy with approximately the same small number of iterations. As we will see in the following, for the optimization of the Jastrow (Jastrow and SDBCS) it is particularly convenient to use $\beta=-1(\beta=0)$, so that $B=S_{h}+\mu S\left(B=S_{h}+G+\mu S\right)$, with $\mu$ determined by (11) when different from zero. Henceforth the $\beta=1$ technique is indicated by NM, namely the standard Newton method used in Refs. (6 12), whereas the one defined by an appropriate choice of the parameter $\beta \neq 1$, is named SR with Hessian acceleration (SRH).

Statistical averages in VMC. In VMC the statistical averages required for each iteration are evaluated over $M$ sampled configurations $\left\{x_{i}\right\}$-named bin-, obtained after a small equilibration, and distributed according to the square of the WF. We indicate the corresponding statistical averages with the symbol $\langle<\rangle\rangle$, e.g. $<<O_{k}(x)>>=1 / M \sum_{i} O_{k}\left(x_{i}\right)$, so that for large $M$ $<<O_{k}(x)>>$ coincides with the exact quantum average $\left\langle O_{k}\right\rangle$, with a statistical accuracy $\propto \frac{1}{\sqrt{M}}$.

After each bin we change the variational parameters according to Eq. (8), by using the appropriate matrix $B$ discussed before. The SRH is stable for small enough $r$ and may converge much faster than SR. With a larger bin length $M$, the value of $r$ can be substantially increased, leading therefore to a much faster convergence within the SRH minimization scheme.

As also noted in Ref $[\underline{6}$ it is extremely important that the quantities evaluated within the statistical approach are obtained by means of fluctuations $\delta A(x)=$ $A(x)-<<A(x)>>$ of suitable variables $A(x)$, that depend only on the electronic configuration $x$ sampled with VMC. Therefore, it is useful to express the forces $f_{k}$, the matrices $S_{h}$ and $S$ by appropriate fluctuation averages:

$$
\begin{aligned}
f_{k} & =-2<<\delta e_{L}(x) \delta O_{k}(x)>> \\
S_{h}^{k, k^{\prime}} & =<<\delta \partial_{\alpha_{k}} e_{L}(x) \delta O_{k^{\prime}}(x)>>+\left(k \leftrightarrow k^{\prime}\right) \\
S^{k, k^{\prime}} & =<<\delta O_{k}(x) \delta O_{k^{\prime}}(x)>> \\
G^{k, k^{\prime}} & =2<<\delta e_{L}(x) \delta O_{k}(x) \delta O_{k^{\prime}}(x)>>
\end{aligned}
$$

where $e_{L}(x)=\frac{\left\langle\psi_{\alpha}|H| x\right\rangle}{\left\langle\psi_{\alpha} \mid x\right\rangle}$ is the so called local energy. In order to derive Eq. (13) we insert a completeness $\sum_{x}|x\rangle\langle x|=I$ in the RHS of Eqs.([6), and we also make use of the identity $\left\langle x\left|H O_{k}\right| \psi_{\alpha}\right\rangle=$ $\left(\partial_{\alpha_{k}} e_{L}(x)+O_{k}(x) e_{L}(x)\right) \psi_{\alpha}(x)$. Then we notice that 
$<<\partial_{\alpha_{k}} e_{L}(x)>>=0$ for $M \rightarrow \infty, \frac{13}{13}$ so that the fluctuations of $\partial_{\alpha_{k}} e_{L}(x)$ can conveniently appear in Eq.(13). This way to evaluate $S_{h}$ is affected by much smaller statistical errors than the previous estimate 12 $<<\partial_{\alpha_{k}} e_{L}(x) O_{k^{\prime}}(x)>>+\left(k \leftrightarrow k^{\prime}\right)$. For the same reason the matrix $B$, used in the iteration (8), is affected by the large noise present in $G$. Indeed, for $\beta=-1$, $B$ does not depend on $G$ and the corresponding statistical fluctuations are much reduced, especially for a large number of electrons $N_{e}$. In fact the operators $O_{k}$ and the local energy scale with $N_{e}$, their fluctuations $\delta e_{L}, \delta O_{k}$ in (15) with $\sqrt{N_{e}}$, whereas the corresponding statistical averages for $G$ are of order $N_{e}$, because they contribute to an extensive energy in (5). This implies zero signal to noise ratio $\left(\simeq \frac{1}{\sqrt{N_{e}}}\right)$ in the matrix $G$ (or similarly in $S_{h}$ without using the correlation 131) for large $N_{e}$ compromising the efficiency of the VMC. On the other hand it is clear that both the matrices $S$ and $S_{h}$ in Eqs. (13) 14) are much better behaved, because they are obtained by averaging statistically quantities of order $N_{e}$, that have a mean value of the same order.

Unfortunately, for the optimization of $|D\rangle$, uncontrolled divergences in the evaluation of $S_{h}^{k, k^{\prime}}$ appear when both indexes $k$ and $k^{\prime}$ correspond to the SDBCS parameters. This is due to the zeros of $\langle x \mid D\rangle$, implying that $\partial_{\alpha_{k}} e_{L}(x) \simeq-O_{k}(x) e_{L}(x)$ and $O_{k^{\prime}}(x)$ wildly fluctuate in Eq.(13) because they can both diverge for $\psi_{\alpha}(x) \rightarrow 0$. The way to overcome this difficulty is to use $\beta=0$ in Eq.(9) since the most relevant divergences in $S_{h}$ are exactly canceled by $G$ just for this value of $\beta$. In principle the matrix $B$ cannot be evaluated statistically for $\beta \neq 0$ because the statistical uncertainty remains even for $M \rightarrow \infty$, due to these large fluctuations. For $\beta=0$ and for large number of electrons $N_{e}$, the problem of zero signal to noise ratio in $B$ cannot be avoided for Jastrow and SDBCS optimization, but can be substantially alleviated by a good WF choice, because $G$ has the zero variance property: $G=0$ without noise, whenever $\psi_{\alpha}$ is an exact eigenstate.

Results We have tested the simple SR method, the presently discussed SRH one, and the recently introduced $\mathrm{NM}^{\underline{6}}$ ( $\beta=1$ in our notations) on simple $L$-sites lattice models, the 1D Heisenberg model (1DHM) and the t-J mode ${ }^{14}$, because we believe they represent useful benchmarks to compare various techniques. The Jastrow factor of the initial WF was set to zero for both models. Moreover, consecutive configurations in each bin are separated by $2 \times L$ Metropolis attempts.

For the $1 \mathrm{DHM}$ we use a WF containing a long range Jastrow factor in the form: $\exp \left(1 / 2 \sum_{i, j} v_{i, j}^{z} S_{i}^{z} S_{j}^{z}\right) \mid D>$ where $|D\rangle$ is a Néel state with magnetization along the $x$-spin axis. Here $\vec{S}$ are usual spin- $1 / 2$ operators and projection over zero total spin $-z$ component is also assumed. The long range Jastrow is essential to destroy the long range magnetic order in $1 \mathrm{D}$ and obtain a sensible and accurate ansatz ${ }^{15}$. By using all spatial symmetries of the model, we remain with $L / 2-1$ independent

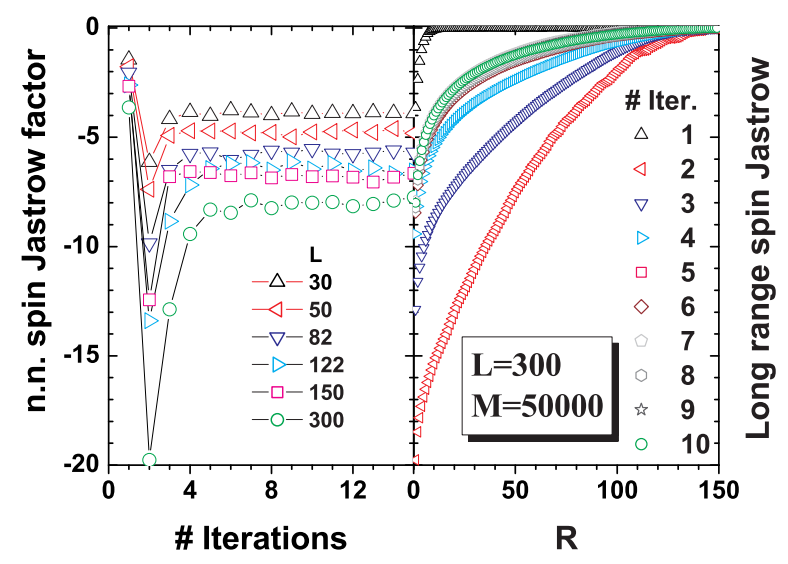

FIG. 1: Left: convergence of the SRH technique for different sizes. Right: the same for all the 149 independent VMC parameters for the maximum size studied. For all simulations the bin length (the control parameter $r$ defined in Eq.(11) $M$ was taken proportional to $L^{2}(L)$, starting from $M=500$ $(r=2)$ for $L=30$.

parameters in the Jastrow factor.

As shown in Fig.(1), with the present SRH technique, a converged value of the nearest neighbor $v^{z}$ is obtained in less than 10 iterations for all sizes studied (similar convergence is obtained at all distances, see right panel), implying that the use of the matrix $S_{h}(\beta=-1$ has been used in this case) is very effective to accelerate the convergence to the minimum energy WF. By the SR technique, stability forces a very slow convergence: the required number of iterations increases with the system size $\propto L^{2}$ because the model is gapless and accurate calculations become prohibitive already for $L \simeq 100$.

As shown in Fig. (2) SRH is remarkably faster than SR. After convergence, with much longer runs, SR and NM appear $\gtrsim 50$ and $\gtrsim 10$ times less efficient than SRH respectively, for obtaining the variational parameter within a given statistical error, by performing a statistical average, as discussed in Ref 10 . SR has a much larger correlation time and NM is affected by larger fluctuations. The performances of SRH appear always optimal just for $\beta=-1$, especially for large $N_{e}$, due to the argument presented below Eq.(15). This value of $\beta$ allows also to use small bin length (see Fig 2) and to be very efficient far away from convergence.

Optimization of $|D\rangle$. We have also tested the SRH technique in the $2 \mathrm{D} t-J$ model at $J / t=0.4^{14}$ for optimizing the parameters contained in the $D=|B C S\rangle$ where the BCS Hamiltonian defining the $B C S$ state has a gap function $\Delta_{k}=\Delta\left(\cos k_{x}-\cos k_{y}\right)$ and a dispersion band $\epsilon_{k}=-2\left(\cos k_{x}+\cos k_{y}\right)-4 t^{\prime} \cos k_{x} \cos k_{y}-\mu_{0}$, where $\mu_{0}, t^{\prime}$ and $\Delta$ are the three variational parameters, that are optimized together with all the forty (ten) independent ones contained in the density- density Jastrow factor $\exp \left(1 / 2 \sum_{i, j} v_{i, j} n_{i} n_{j}\right)$ for $L=242(L=50)$. In this 


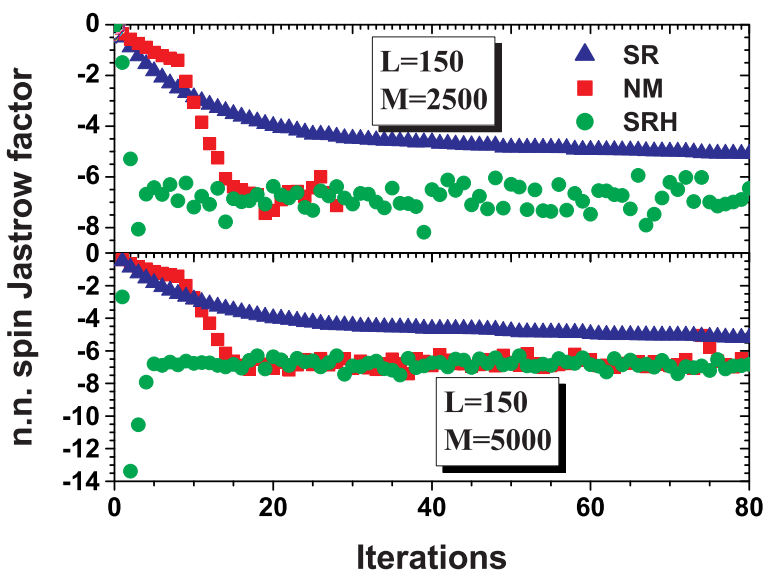

FIG. 2: Convergence of the VMC n.n. spin Jastrow for various methods (see text). For the $\mathrm{SR}$ technique $\Delta t J=$ 0.125 . For the NM the first 4 iterations are with $a_{\text {diag }}=0.02$, for the next $6 a_{\text {diag }}=0.002$ and the rest with $a_{\text {diag }}=0$. For $M=2500$ the NM is unstable after 30 iterations due to a big fluctuation of the matrix $G$ (see text) not used in SRH. For the SRH technique $r=5$ for $M=2500$ and $r=10$ for $M=5000$. After the third iteration $|\Delta \mathrm{WF}|<r$ is always satisfied, even after further 1000 iterations (not shown).

case, as discussed before, $\beta=0$ has to be used, namely $B=S_{h}+G$ in the iteration scheme (8). Moreover, in contrast to the Jastrow case, the condition (11) cannot be always verified with $\mu=0$ after equilibration, due to occasionally large fluctuations in the matrix $B$. Nevertheless, the SRH remains stable, by using a small enough value of $r$. In this way, these large fluctuations of $B$ are very efficiently damped by condition (11). As shown in Fig.(3), also in this case the SRH provides a substantial reduction of the iterations required for convergence. As expected this is accompanied also by a sizable increase of the fluctuations in the parameters especially for the chemical potential $\mu_{0}$. Indeed, though the efficiency increases with $L$, in favor of SRH. this improvement is not as important as in the previous case, for the optimization of the Jastrow part alone.

For the simultaneous optimization of the Jastrow and SDBCS parts of the WF, a more efficient scheme is to use the $\mathrm{SR}$ for the SDBCS parameters and the $\mathrm{SRH}$ with $\beta=$ -1 for the remaining ones (hybrid method in Fig [3). In this way, in the iteration (8), the noisy and computationally expensive ( $\simeq 5$ times more) SDBCS matrix elements $S_{h}^{k, k^{\prime}}$ are replaced by $S^{k, k^{\prime}} / \Delta t$, whereas all the other matrix elements of $S_{h}$ are evaluated including also the ones coupling $J$ and $|D\rangle$. For these matrix elements an estimator different from the symmetric one (13) is used for $S_{h}: S_{h}^{k, k^{\prime}}=S_{h}^{k^{\prime}, k}=2<<\delta \partial_{\alpha_{k}} e_{L}(x) \delta O_{k^{\prime}}(x)>>$, involving only local energy derivatives of Jastrow parameters. Afterall suitable values of $r$ and $\Delta t$ provide performances similar to $\mathrm{SRH}$ for the size studied, with a much cheaper

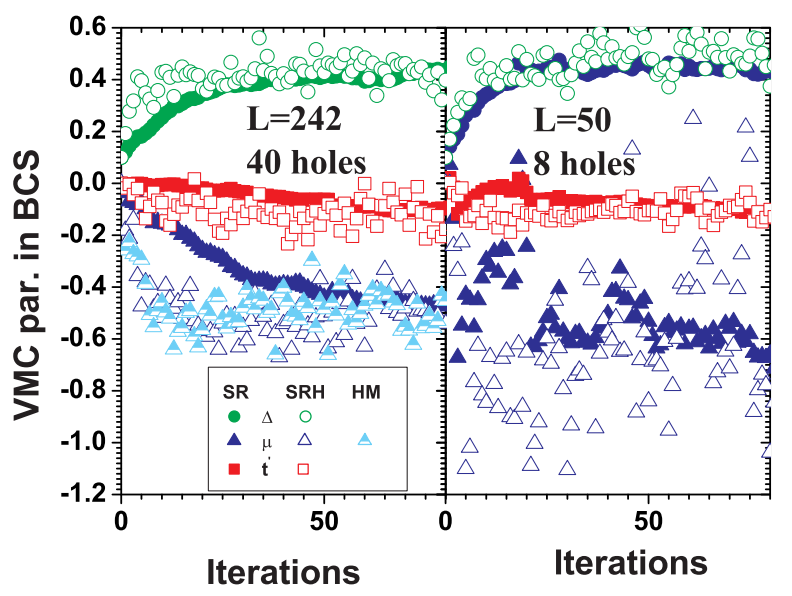

FIG. 3: Parameters in $|D\rangle$, while optimizing the Jastrow part simultaneously. for the $t-J$ model at $J / t=0.4$ with bin $M=$ 5000. For SRH the control parameter is $r=0.5(r=0.1)$ for $L=242(L=50)$, for SR $J \Delta t=0.125$. The hybrid method (HM) is also shown (left) for $\mu_{0}(r=1 J \Delta t=1.25)$.

computational cost per iteration and with a signal to noise ratio certainly stable for large $N_{e}$, as only $S^{h}$ and $S$ (and not $G$ ) are used in this case.

In conclusion, the optimization methods we have described, based on a very efficient VMC evaluation of the Hessian matrix, open the possibility to study electronic systems with correlated WF containing many variational parameters, and with an efficiency that is now comparable with non statistical methods of some time ago 16 .

I acknowledge useful comments by A. Parola. M. Casula, F. Becca, C. Filippi, and C. Umrigar. This work was partially supported by COFIN MIUR-2003.
1 R. B. Laughlin, Phys. Rev. Lett. 50, 1395 (1983).

2 P. W. Anderson, Science 235, 1196 (1987).

${ }^{3}$ G. Dev, J. K. Jain Phys. Rev. B 45, 1223 (1992).

4 C. Gros Phys. Rev. B 38, R931 (1988).

5 T. Giamarchi, C. Lhuillier Phys. Rev. B 43, 12943 (1991).

${ }^{6}$ C. J. Umrigar, C. Filippi to appear in Phys. Rev. Lett. .

7 F. Schautz, C. Filippi J. Chem. Phys. 120, 10931 (2004).
8 A. Harju et al., Phys. Rev. Lett. 79, 1173 (1997).

9 M. Capello et al., Phys. Rev. Lett. 94, 026406 (2005).

10 M. Casula, C. Attaccalite, S. Sorella J. Chem. Phys. 121, 7110 (2004).

11 When the lowest eigenvalue of $S$ is very small, for a stable method, the irrelevant parameters of the WF have to be removed, e.g. with the method described in Ref 10. 
12 M. Casalegno, M. Mella, A. M. Rappe, J. Chem. Phys. 118, 7193 (2003).

13 R. Assaraf, M. Caffarel Phys. Rev. Lett. 83, 4682 (1999).

14 S. Sorella et al. Phys. Rev. Lett. 88, 117002 (2002).
15 see e.g. E. Manousakis, Rev. Mod. Phys. 63, 1 (1991).

16 R. Car and M. Parrinello Phys. Rev. Lett. 55, 2471 (1985). 